\title{
Sex Differences in Cardiac AMP-Activated Protein Kinase Following Exhaustive Exercise
}

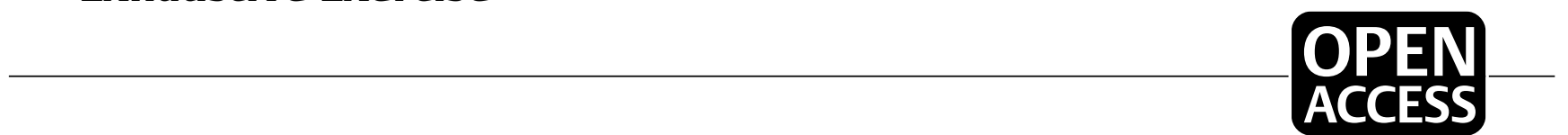

\section{(ㄷ) (1)}

\author{
Authors \\ Kevin D. Brown ${ }^{{ }^{*}}$, Edward D. Waggy ${ }^{{ }^{*}}$, Sreejayan Nair ${ }^{2,3}$ (D), Timothy J. Robinson ${ }^{3}$, Emily E. Schmitt ${ }^{1,3}$, Danielle R. Bruns ${ }^{1,3}$,
} D. Paul Thomas ${ }^{1}$

\section{Affiliations}

1 Division of Kinesiology \& Health, University of Wyoming, College of Health Sciences, Laramie, United States

2 School of Pharmacy, University of Wyoming, Laramie, United States

3 WWAMI Medical Education, University of Wyoming, Laramie, United States

Keywords

sex differences, cardiac, exercise, AMPK

received $\quad 23.12 .2019$

revised $\quad 25.01 .2020$

accepted $\quad 05.02 .2020$

Bibliography

DOI https://doi.org/10.1055/a-1115-6373

Sports Medicine International Open 2020; 4: E13-E18

(c) Georg Thieme Verlag KG Stuttgart · New York

ISSN 2367-1890

Correspondence

Dr. Danielle R. Bruns

Department of Kinesiology \& Health, University of Wyoming 1000 E University Ave

82071-2000 Laramie

United States

Tel.: + 1307766 5290, Fax: + 13077664098

dbruns1@uwyo.edu

\section{ABSTRACT}

Ischemic heart disease presents with significant differences between sexes. Endurance exercise protects the heart against ischemic disease and also distinctly impacts male and female patients through unidentified mechanisms, though some evidence implicates 5 '-AMP-activated protein kinase (AMPK). The purpose of this investigation was to assess the impact of training and sex on cardiac AMPK activation following exhaustive exercise. AMPK activation was measured in trained and sedentary mice of both sexes. Trained mice ran on a treadmill at progressively increasing speeds and duration for 12 weeks. Trained and sedentary mice of both sexes were euthanized immediately following exhaustive exercise and compared to sedentary controls. Endurance training elicited adaptations indicative of aerobic adaptation including higher max running velocities and cardiac hypertrophy with no differences between males and females. AMPK activity was higher in male compared to females, and trained exhibited higher AMPK activity compared to sedentary mice. In response to training, male mice activated AMPK more robustly than female mice. Chronic exercise training increases the ability to activate cardiac AMPK in response to exhaustive exercise in a sex-specific manner. Understanding the interaction between exercise and sex is vital for use of exercise as medicine for heart disease in both men and women.

\section{Introduction}

Sex differences underlie the physiology, pathophysiology, as well as social and economic responses to several human diseases, including cardiovascular disease [1]. Ischemic heart disease, the leading cause of death in the US [2], is one of such diseases characterized by significant differences between biological sexes. Although many factors likely contribute to these significant sex differences including access to healthcare, and social and economic circum-

* co-first authors, contributed equally to the publication stances [3], a wealth of data also suggests that biological and physiological factors result in different pathogenesis and treatment outcomes in males compared to females [4]. Regular endurance exercise is the most cardioprotective intervention yet identified [5] and elicits significant differences between sexes. Given the importance of exercise in cardiovascular disease, it is imperative that we understand the sex-specific mechanisms by which exercise protects the heart. 5'-AMP-activated protein kinase (AMPK) is a key regulator of energy balance in the heart and has recently emerged as a target for cardioprotection [6, 7]. AMPK activation by a single bout of 
exercise and exercise training both reduce infarct size [8, 9], highlighting the critical role of cardiac AMPK in exercise-mediated protection. Though reports of sex differences in AMPK exist in skeletal muscle in response to endurance exercise [10], sex-specific regulation of AMPK in the heart has not been previously examined in response to exhaustive exercise and training. Thus, the purpose of the current investigation was to determine the impact of sex and endurance training on cardiac AMPK activation in response to exhaustive exercise.

\section{Materials and Methods}

We confirm that this study meets the ethical standards of the journal [11].

\section{Animal care}

All methods described in this experiment were approved by the Institutional Animal Care Users Committee protocol \# A-3216-10. Healthy male and female C57BL6 mice 13-16 weeks old and weighing 20-25g were randomly assigned to an experimental group, after which they were caged with their litter mates. Cages were housed in a temperature-controlled room $\left(21^{\circ} \mathrm{C}\right)$ with 12 :12-hour light/dark cycle. Animals were given ad libitum access to fresh water and Purina ${ }^{\circledR}$ brand Laboratory Rodent Chow.

\section{Research design}

Mice were randomly assigned to one of three groups: sedentaryresting controls (SED-Rest; males $n=5$; females $n=5$ ), sedentaryexhausted (SED-Exhausted; males $n=9$; females $n=8$ ), and trainedexhausted (TR-Exhausted; males $n=10$; females $n=10$ ). SED-Rest mice received no exercise training and were sacrificed in a resting state after acclimation to light handling. SED-Exhausted mice were acclimated to a Stanhope rodent treadmill at slow speeds $(<10 \mathrm{~m} /$ min, $10 \mathrm{~min} /$ day) for one week. TR-Exhausted mice were subjected to 12 weeks of endurance training as described below. TR-Exhausted and SED-Exhausted animals were sacrificed immediately after a maximal exhaustive bout of exercise that occurred $24 \mathrm{~h}$ after their last training bout.

\section{Endurance exercise training protocol}

TR-exhausted mice began acclimating to treadmill exercise for the first five days of the protocol (days $0-4$ ) and started training on day 6 . Animals in the TR-Exhausted group were exercised six days per week for a total of 12 + weeks. Training sessions took place between 18:00 and 21:00, because this was the beginning of the animal's dark cycle. The incline remained at $25^{\circ}$, and speed and duration were gradually increased until animals could maintain $22 \mathrm{~m} / \mathrm{min}$ for $60 \mathrm{~min} /$ day (days 36-41). This speed and duration were sustained for the remainder of the training period ( $\vee$ Fig. 1a)

\section{Exhaustive exercise test}

An exhaustive exercise test was performed using a modified graded protocol as previously published [12,13]. Briefly, the test began at $15 \mathrm{~m} / \mathrm{min}$ for the SED group and $20 \mathrm{~m} / \mathrm{min}$ for TR group. Treadmill speed was increased by $4 \mathrm{~m} / \mathrm{min}$ every $3 \mathrm{~min}$. Once at $40 \mathrm{~m} /$ $\mathrm{min}$, the speed increased by $2 \mathrm{~m} / \mathrm{min}$ every $2 \mathrm{~min}$. Termination occurred when the animal could no longer keep pace with the treadmill and ceased to run. Sacrifice took place immediately post-exercise. TR-Exhausted animals completed the exhaustive protocol $24 \mathrm{~h}$ after their last training regimen. SED-Exhausted mice were tested $24 \mathrm{~h}$ after their last treadmill acclimatization session. SED-Rest controls were sacrificed in a rested state. The exhaustive exercise test is summarized in $>$ Fig. $\mathbf{1}$ b.

\section{Tissue extraction, homogenization, immunoblotting}

Tissue samples were collected from the apex of the left ventricle (LV), as well as from the remaining portion of the LV and right ventricle ("whole heart"). All animals were sacrificed via cervical dislo- a

\begin{tabular}{|c|c|c|}
\hline Days & Speed (m/min.) & Time (min.) \\
\hline $1-3$ & 8 & 15 \\
\hline $4,5,7$ & 10 & 20 \\
\hline 8 & 10 & 25 \\
\hline 9,10 & 12 & 25 \\
\hline 11,12 & 12 & 30 \\
\hline 14 & 14 & 30 \\
\hline 15,16 & 14 & 35 \\
\hline 17 & 16 & 35 \\
\hline 18,19 & 16 & 40 \\
\hline 21 & 18 & 40 \\
\hline 22,23 & 18 & 45 \\
\hline 24 & 20 & 45 \\
\hline 25,26 & 20 & 50 \\
\hline $28-30$ & 22 & 50 \\
\hline $31-33,35,36$ & 22 & 55 \\
\hline$* 36-89$ & 22 & 60 \\
\hline
\end{tabular}

b

\begin{tabular}{|c|c|c|}
\hline & SED & TR \\
\hline Start & 15 & 20 \\
\hline 3 min & 19 & 24 \\
\hline 6 min & 23 & 28 \\
\hline 9 min & 27 & 32 \\
\hline 12 min & 31 & 36 \\
\hline 16 min & 35 & 40 \\
\hline 18 min & ---- & 42 \\
\hline 19 min & 38 & 44 \\
\hline 21 min & 40 & 46 \\
\hline 23 min & 42 & 48 \\
\hline
\end{tabular}

Fig. 1 Training and exhaustive exercise bout protocols. a Summary of 12-week training protocol. b Summary of exhaustion protocol for trained (TR) and sedentary (SED) exhausted mice. 
cation. Hearts were rapidly excised, dissected, and freeze-clamped at liquid nitrogen temperatures. Tissue samples were weighed, mechanically homogenized in an ice-cold lysis buffer (20 mM HEPES $\mathrm{pH}$ 7.4, 50 mM $\beta$-glycerol phosphate, 2 mM EGTA, 1 mM DTT, $10 \mathrm{mM} \mathrm{NaF}, 1 \mathrm{mM}$ sodium orthovanadate, $1 \%$ triton- $100,10 \%$ glycerol, protease inhibitor cocktail), centrifuged at $10000 \mathrm{~g}$ for $10 \mathrm{~min}$ at $4{ }^{\circ} \mathrm{C}$. A micro BCA protein assay kit was used to determine protein concentration.

AMPK expression was measured using standard immunoblotting techniques. Briefly, samples were loaded on a $10 \%$ polyacrylamide gel and separated by sodium dodecyl sulfate-polyacrylamide gel electrophoresis. Proteins were transferred to polyvinylidene difluoride membranes and blocked in tris-buffered saline and $5 \%$ nonfat milk for $1 \mathrm{~h}$ at room temperature. The membranes were incubated overnight at $4{ }^{\circ} \mathrm{C}$ with primary antibodies (1:1000 dilution) specific to either total AMPK or phosphorylation of AMPK $\alpha$ at $\mathrm{Thr}^{172}$. Membranes were incubated in secondary anti-rabbit antibodies $(1: 1000)$ for $1 \mathrm{~h}$ at room temperature, exposed to chemiluminescence reagents and imaged using autoradiography. Analysis of protein bands was quantified using Image].

\section{AMPK activity}

Apex cell lysates were used for the AMPK activity assay. AMPK activity was determined using a ${ }^{32} \mathrm{P}$ radioimmunoassay with SAMS (His-Met-Arg-Ser-A\&-Met-Ser-Gly-Leu- His-Leu-Val-Lys- Arg- Arg) as a substrate as previously described $[14,15]$. Briefly, LV apex AMPK activity was calculated by nanomoles of ATP incorporated into the SAMS peptide/gram of muscle per minute.

\section{Statistical analysis}

Body weight was compared by a repeated measures 2-way ANOVA (sex $\times$ training). A 2-way ANOVA (sex $\times$ training) with a Tukey post hoc test was used to compare all other outcome variables. In each case, the independent effects of sex and training are reported with post-hoc comparisons. A multiple linear regression model was used to investigate the impact of sex and training on the correlation between heart weight, AMPK activity, and max running speed using $R$ statistical software. All other data were analyzed using JMP Version $14^{\odot}$ statistical software (JMP, Cary, NC, USA). $P<0.05$ was considered indicative of statistical significance and all data were expressed as means \pm SEM.

\section{Results}

\section{Baseline animal characteristics}

Forty-eight C57BL/6 mice (males $n=24$; females $n=24$ ) at 15-19 weeks were used in this study. A total of 20 mice were assigned to the TR-Exhausted group (males $n=10$; females $n=10$ ), 17 to the SED-Exhausted group (males $n=9$; females $n=8$ ), and 11 (males $n=5$; females $n=6$ ) to the SED-Rest group to serve as non-exercised, non-exhausted controls. All animals were considered healthy as none displayed signs or symptoms of illness or disease. As expected, male mice had significantly higher body weights than fe- male mice. SED and TR had similar body weights at the beginning of the training protocol.

\section{Indicators of training adaptation}

Animals (male and female) in the TR-Exhausted groups maintained body weight throughout the study, gaining $0.31 \pm 0.53 \mathrm{~g}$, whereas the SED-Exhausted groups gained $3.28 \pm 0.57 \mathrm{~g}$, and the SED-Rest controls gained $3.78 \pm 0.71 \mathrm{~g}$, a significant effect of training. Interestingly, male mice in the TR-Exhausted group lost weight over the 12-week training period, whereas female mice did not ( $>$ Fig. 2a).

TR-Exhausted animals had significantly higher (25\%) maximal running velocity compared to SED-Exhausted animals ( $44 \pm 0.43$ vs. $33 \pm 0.68 \mathrm{~m} / \mathrm{min}$; Fig. 2b). TR-Exhausted animals had a significantly larger heart relative to body weight ( $\mathrm{Ht} / \mathrm{BW} ; 5.1 \pm 0.13 \mathrm{mg} / \mathrm{g}$ ) compared to SED-Exhausted $(4.5 \pm 0.16 \mathrm{mg} / \mathrm{g})$ and SED-Resting animals ( $4.1 \pm 0.11 \mathrm{mg} / \mathrm{g}$; $>$ Fig. $2 \mathrm{c})$. Ht/BW was not influenced by sex. We also normalized heart weight to max running velocity ( $\triangleright$ Fig. 2d). Although we found a significant effect of training, there was no impact of sex and no interaction between sex and training.

\section{AMPK activity by sex and training}

Cardiac AMPK activity was significantly greater in male mice compared to females and in TR compared to SED. Post-hoc analyses indicated that both sexes activated cardiac AMPK activity after a bout of exhaustive exercise ( $>$ Fig. 3a). Sex and training differences in cardiac AMPK activity were likewise found in phospho/total AMPK expression, with a significant effect of sex and training status and higher activation of AMPK in TR-Exhausted male than female mice ( Fig. 3b).

\section{Correlations between indicators of training adaption and cardiac AMPK activity}

We performed multiple linear regressions to determine whether there were significant associations between indicators of training adaption and cardiac AMPK activity and how these associations differed by sex and training status. There was a positive correlation between $\mathrm{Ht} / \mathrm{BW}$ and max running speed ( $>$ Fig. $\mathbf{4 a}$ ). AMPK activity correlated with max running speed in male mice but not in female mice ( $>$ Fig. 4b). There was no significant linear association between AMPK activity and Ht/BW in either sex ( $\mathbf{F i g}$. 4c). Together, these associations suggest that males demonstrate higher cardiac AMPK activity relative to aerobic adaptation.

\section{Discussion}

The purpose of this study was to investigate the effect of endurance exercise training on cardiac AMPK activity in male and female C56BL/6 mice after an exhaustive bout of exercise. The primary goal was to assess sex differences in cardiac AMPK activity resulting from an acute exercise bout as previously found in human skeletal muscle [10]. We found striking fundamental differences in how male and female mice respond to cardiometabolic stress. Regardless of training status, male mice have significantly higher levels of cardiac AMPK activity in response to an acute bout of exhaustive exercise compared to female mice. We also show that trained mice have a greater capacity to activate cardiac AMPK in response to in- 

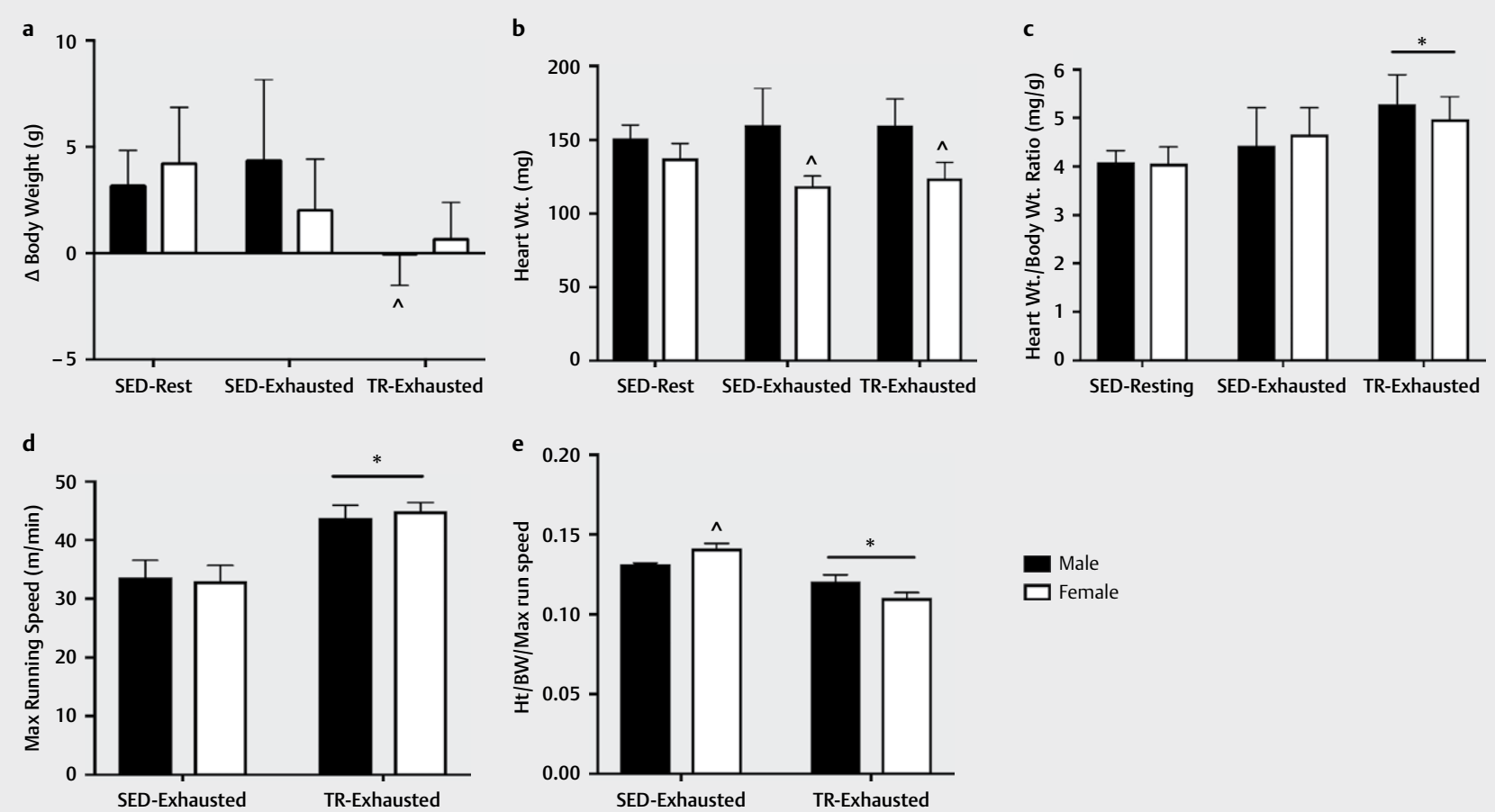

- Fig. 2 Training adaptations and cardiac morphometrics for male and female trained (TR) and sedentary (SED) rest and exhausted mice. a Body weight changes in male and female mice. $\mathbf{b}$ Heart weight in male and female mice. $\mathbf{c}$ Heart/body weight. $\mathbf{d}$ Max running speed. $\mathbf{e}$ Heart weight normalized to training (max running speed). Data were analyzed by a 2-way ANOVA with sex and training as independent variables. $n=20$ TR-Exhausted (10 male, 10 female); $n=18$ SED-Exhausted ( 9 male, 9 female); and $n=10$ SED-Rest ( 5 male, 5 female). ${ }^{*} p<0.05$ effect of training, ${ }^{\wedge} p<0.05$ significant effect of sex. Data are presented as means \pm SEM.

creased metabolic demand, potentially because they were able to achieve higher workloads, thus necessitating greater AMPK activation, although this activation also differed by sex. Consistent with the hypothesis that greater demand necessitates greater AMPK activation, AMPK activity is suggested to be activated in a graded fashion by exercise, with higher workloads eliciting greater activity [16]. It is not known if higher capacity to activate AMPK in trained mice is a requirement for higher maximal running velocity, or vice versa. However, our data add novelty to the existing body of work, demonstrating that despite similar exercise intensity (max running speed) and cardiac remodeling (hypertrophy), male mice still demonstrate higher AMPK activity than females.

Our primary finding of sex-specific activation of cardiac AMPK in response to exhaustive exercise is interesting in light of clinical findings demonstrating sex-specific outcomes in males and females with heart disease. Our data align with one previous report in which three weeks of voluntary wheel running increased cardiac PAMPK expression in male but not female mice [17]. Basal AMPK expression differences between sexes have been reported to remain into older age, with 22-month old mice demonstrating differential expression in the kidney and brain but not in the liver, also suggesting tissue-specific regulation of AMPK by sex [18]. Clinically, how activation of AMPK may be manipulated to inform disease outcomes in a sex-specific manner remains understudied. However, our data raise the question whether AMPK activators may protect males from ischemic disease more effectively than females. Continued sex and tissue-specific studies of the impact of both exercise on males and females are warranted to tailor pharmacological or lifestyle approaches for human medicine.

An exciting area of research regarding the effect of exercise on cardioprotection involves the ability of exercise training to stimulate expression of myocardial ATP-sensitive $\mathrm{K}+\left(\mathrm{K}_{\text {ATP }}\right)$ channels. Sex differences have been observed in the rat heart concerning these $\mathrm{K}_{\text {ATP }}$ channels, with sedentary females showing significantly higher expression of both subunits. This has been correlated to significantly lower infarct size in female rats (25\%) compared to males (37\%) [19]. Additional downstream AMPK targets might demonstrate sexspecific outcomes Similar to downstream targets of AMPK, the mechanisms upstream responsible for sex-specific regulation of AMPK remain somewhat unclear. Estrogen (E2) is reported to activate AMPK through the estrogen receptor $\alpha(E R \alpha)$, via phosphorylation of Thr172 of the $\alpha$-catalytic subunit [20]. However, the interplay between exercise, AMPK, and estrogen signaling has not been fully described.

We also found differences between males and females with regard to endurance training. Interestingly, whereas male mice lost body weight over the 12-week training period, female mice did not, consistent with epidemiological data in women undergoing exercise-induced weight loss programs [21]. Previous reports of sex dif- 

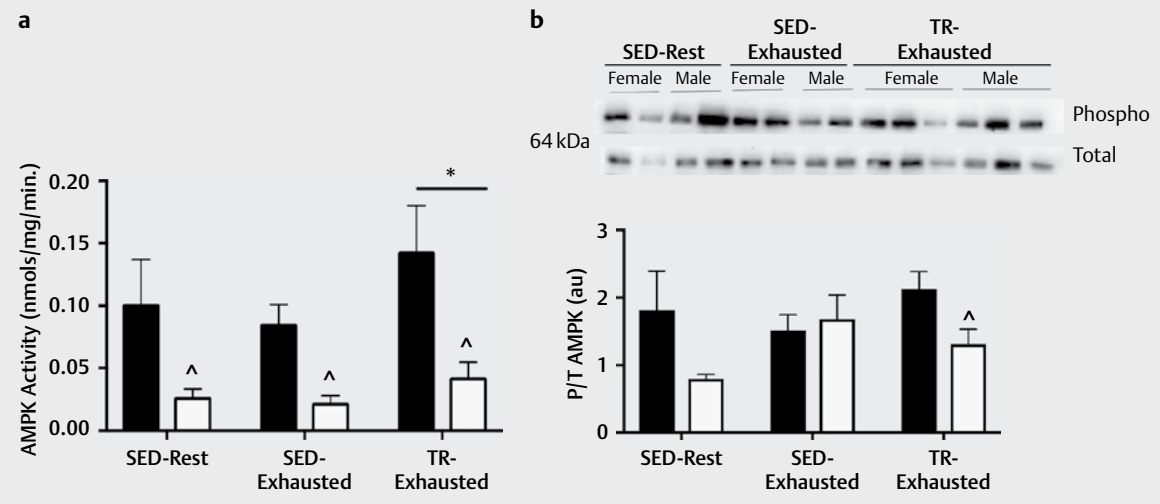

- Male $\square$ Female

- Fig. 3 Left ventricular AMPK activation in trained-exhausted (TR-Exhausted), sedentary-exhausted (SED-Exhausted), and sedentary-resting (SEDRest) groups for both male and female mice. a LV AMPK activity. $n=20$ TR-Exhausted (10 male, 10 female); SED-Exhausted ( 8 male, 8 female); SEDRest (5 male, 5 female). ${ }^{*} p<0.05$ significant effect of training, ${ }^{\wedge} p<0.05$ significant effect of sex, \#significant difference within sex. $\mathbf{b}$ LV AMPK protein expression with representative image. $n=20$ TR-Exhausted ( 6 male, 6 female), SED-Exhausted ( 4 male, 4 female), SED-Rest ( 4 male, 4 female). ${ }^{*} p<0.05$ significant effect of training, ${ }^{\wedge} p<0.05$ significant effect of sex, \#significant difference within sex. Data were analyzed by 2 -way ANOVA (sex $\times$ training) with post hoc analyses. Data are presented as means \pm SEM.
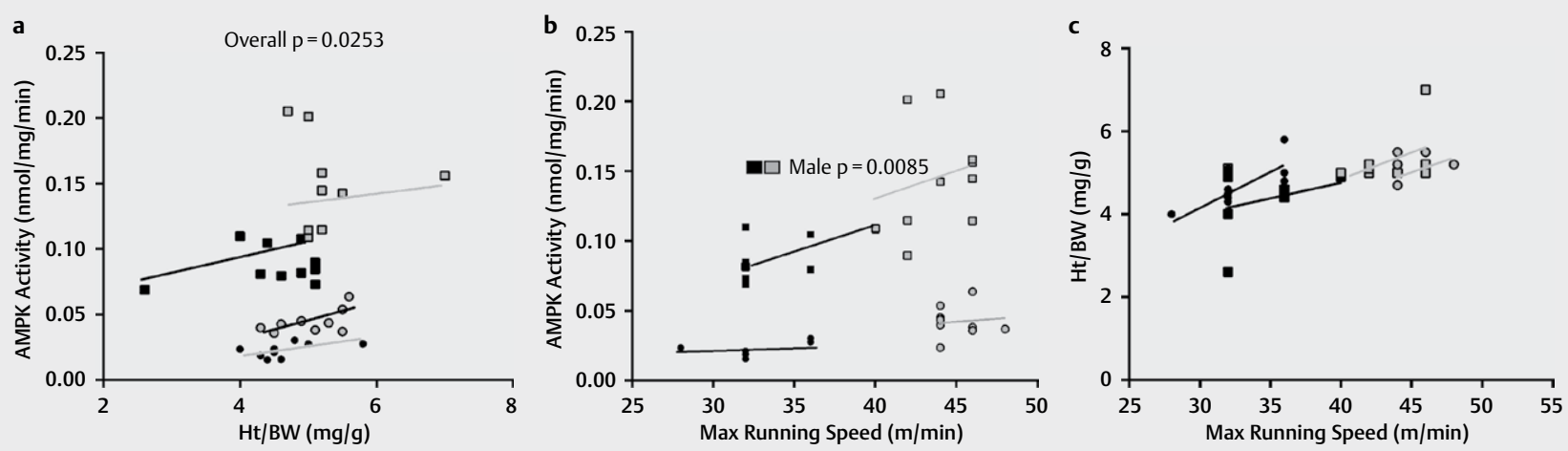

- TR-Exhausted — SED-Exhausted a Male $\bullet$ Female

- Fig. 4 Linear association between cardiac AMPK activity and indicators of aerobic training in male and female mice. a Heart weight normalized to body weight and max running speed. $\mathbf{b}$ AMPK activity and max running speed. c AMPK activity and heart weight normalized to body weight. Data were analyzed by multiple linear regressions. $n=20$ TR-Exhausted ( 10 male, 10 female); $n=18$ SED-Exhausted ( 9 male, 9 female); and $n=10$ SED-Rest (5 male, 5 female).

ferences in endurance training report greater cardiac hypertrophy in female mice than males [17]. However, these investigations utilized free wheel-running whereas our study used forced treadmill training. Indicators of endurance training adaption suggest that our trained mice underwent sufficient training, because we report higher maximal running velocities as well as higher heart weight/ body weight for both sexes. Further, previous work from our group reports elevated citrate synthase activity in the quadriceps femoris of mice who underwent a similar training protocol [12]. Thus perhaps differences (physiological, psychological) exist between forced and voluntary exercise that explain the differences between males and females in our data. Future work should elucidate the impact of sex on these different exercise modalities.

\section{Limitations and Conclusions}

The current work did not differentiate cardiac AMPK subunits. AMPK is composed of two catalytic subunits, $\alpha 1$ and $\alpha 2$, with AMPK $\alpha 2$ being the predominant catalytic subunit in the myocardium. Previous reports suggest that AMPK $\alpha 2$ is also the predominant isoform activated following endurance exercise training in the heart [22]. Although we were unable to assess isoform specific activation in the current study, unpublished data from our lab suggests that male TR-Exhausted mice have pronounced AMPK $\alpha 2$ activation. Whether this remains true in female mice is unknown, and 
isoform-specific investigations in both sexes are warranted. Further studies should also elucidate the impact of age (i. e., do sex differences change across the life course, does genetic background impact sex differences of AMPK activation), and the therapeutic outcomes of AMPK activators and inhibitors in models of both sexes. In conclusion, significant sex differences underlie the pathogenesis of cardiovascular disease and the response to regular endurance exercise. Cardiac activation of AMPK is different between male and female mice. Understanding how the mechanisms up- and downstream of AMPK contribute to sex differences is vital if we are to better treat heart disease in men and women.

\section{Funding}

This study was supported by funding from the College of Health Sciences at the University of Wyoming to KDB and EDW (now deceased, "Ed you will be sorely missed").

\section{Conflict of Interest}

The authors declare that they have no conflict of interest.

\section{References}

[1] Maric-Bilkan C, Arnold AP, Taylor DA et al. Report of the National Heart, Lung, and Blood Institute Working Group on Sex Differences Research in Cardiovascular Disease: Scientific Questions and Challenges. Hypertension 2016; 67: 802-807. doi:10.1161/ HYPERTENSIONAHA.115.06967

[2] Xu ], Kochanek KD, Murphy SL et al. Deaths: Final Data for 2007. National Vital Statistics Reports 2010; 58: 1-135

[3] Mosca L, Hammond G, Mochari-Greenberger $\mathrm{H}$ et al. Fifteen-year trends in awareness of heart disease in women: results of a 2012 American Heart Association national survey. Circulation 2013; 127: 1254-1263 e1251-1229. doi:10.1161/CIR.0b013e318287cf2f

[4] Blenck CL, Harvey PA, Reckelhoff JF et al. The importance of biological sex and estrogen in rodent models of cardiovascular health and disease. Circ Res 2016; 118: 1294-1312. doi:10.1161/CIRCRESAHA.116.307509

[5] Lee DC, Sui X, Artero EG et al. Long-term effects of changes in cardiorespiratory fitness and body mass index on all-cause and cardiovascular disease mortality in men: The Aerobics Center Longitudinal Study. Circulation 2011; 124: 2483-2490. doi:10.1161/ CIRCULATIONAHA.111.038422

[6] Qi D, Young LH. AMPK: energy sensor and survival mechanism in the ischemic heart. Trends Endocrinol Metab 2015; 26: 422-429. doi:10.1016/j.tem.2015.05.010

[7] Russell RR 3rd, Li J, Coven DL et al. AMP-activated protein kinase mediates ischemic glucose uptake and prevents postischemic cardiac dysfunction, apoptosis, and injury. J Clin Invest 2004; 114: 495-503

[8] Kristiansen SB, Solskov L, Jessen $\mathrm{N}$ et al. 5-Aminoimidazole-4carboxamide-1- $\beta$-d-ribofuranoside increases myocardial glucose uptake during reperfusion and induces late pre-conditioning: Potential role of AMP-activated protein kinase. Basic Clin Pharmacol Toxicol 2009; $10-16$

[9] Brown DA, Jew KN, Sparagna GC et al. Exercise training preserves coronary flow and reduces infarct size after ischemia-reperfusion in rat heart. J Appl Physiol 2003; 95: 2510-2518

[10] Roepstorff C, Thiele M, Hillig T et al. Higher skeletal muscle 2AMPK activation and lower energy charge and fat oxidation in men than in women during submaximal exercise. J Physiol 2006; 574: 125-138. doi:10.1113/jphysiol.2006.108720

[11] Harriss DJ, MacSween A, Atkinson G. Ethical standards in sport and exercise science research: 2020 update. Int J Sports Med 2019; 40: 813-817. doi:10.1055/a-1015-3123

[12] Wolff AM, Rasmussen TP, Wichern CR et al. Effects of pericardiectomy on training- and myocardial infarction-induced left ventricular hypertrophy, chamber dimensions and gene expression. Int J Sports Med 2017; 38: 27-34. doi:10.1055/s-0042-115567

[13] Kemi O], Loennechen JP, Wisloff U et al. Intensity-controlled treadmill running in mice: cardiac and skeletal muscle hypertrophy. J Appl Physiol (1985) 2002; 93: 1301-1309. doi:10.1152/japplphysiol.00231.2002

[14] Davies SP, Carling D, Hardie DG. Tissue distribution of the AMP-activated protein kinase, and lack of activation by cyclic-AMP-dependent protein kinase, studied using a specific and sensitive peptide assay. Eur J Biochem 1989; 186: 123-128

[15] Shen QW, Means W], Underwood KR et al. Early post-mortem AMP-activated protein kinase (AMPK) activation leads to phosphofructokinase-2 and -1 (PFK-2 and PFK-1) phosphorylation and the development of pale, soft, and exudative (PSE) conditions in porcine longissimus muscle. J Agric Food Chem 2006; 54: 5583-5589. doi:10.1021/jf060411k

[16] Coven DL, Hu X, Cong L et al. Physiological role of AMP-activated protein kinase in the heart: Graded activation during exercise. Am J Physiol Endocrinol Metab 2003; 285: E629-E636. doi:10.1152/ ajpendo.00171.2003

[17] Konhilas JP, Chen H, Luczak E et al. Diet and sex modify exercise and cardiac adaptation in the mouse. Am J Physiol Heart Circ Physiol 2015; 308: H135-H145. doi:10.1152/ajpheart.00532.2014

[18] Zawada I, Masternak MM, List EO et al. Gene expression of key regulators of mitochondrial biogenesis is sex dependent in mice with growth hormone receptor deletion in liver. Aging (Albany NY) 2015; 7: 195-204. doi:10.18632/aging.100733

[19] Brown DA, Lynch JM, Armstrong C] et al. Susceptibility of the heart to ischaemia-reperfusion injury and exercise-induced cardioprotection are sex-dependent in the rat. J Physiol 2005; 564: 619-630. doi:jphysiol.2004.081323

[20] Lipovka Y, Chen H, Vagner J et al. Oestrogen receptors interact with the alpha-catalytic subunit of AMP-activated protein kinase. Biosci Rep 2015; 35. doi:10.1042/BSR20150074

[21] Williams RL, Wood LG, Collins CE et al. Effectiveness of weight loss interventions - is there a difference between men and women: A systematic review. Obes Rev 2015; 16: 171-186. doi:10.1111/ obr.12241

[22] Musi N, Hirshman MF, Arad M et al. Functional role of AMP-activated protein kinase in the heart during exercise. FEBS Lett 2005; 579: 2045-2050. doi:10.1016/j.febslet.2005.02.052 